\title{
BIOCOMPUESTOS A BASE DE ALMIDÓN TERMOPLÁSTICO, ÁCIDO POLILÁCTICO Y CASCARILLA DE ARROZ: EFECTO DEL ACEITE EPOXIDADO DE SOYA
}

\author{
BIOCOMPOSITES BASED THERMOPLASTIC STARCH, \\ POLILACTIC ACID AND RICE HUSK: \\ EFFECT OF EPOXIDIZED SOYBEAN OIL
}

$\bullet \bullet$

\author{
Rodrigo Ortega Toro \\ Universidad de Cartagena, Facultad de Ingenieria, Cartagena, Colombia \\ rortegap1@unicartagena.edu.co, orcid.org/0000-0003-0815-5317
}

Recibido: 15/08/2016 - Aprobado: 30/08/2016

\section{RESUMEN}

En Colombia se generan cantidades enormes de subproductos lignocelulósicos, la mayoría es incinerada ylo depositado en el suelo. El objetivo del presente trabajo fue estudiar el desarrollo de materiales compuestos a base de almidón termoplástico (TPS), Ácido Poliláctico (PLA), cascarilla de arroz (RH) y aceite epoxidado de soja (ESO) como una alternativa al uso RH. Se hicieron las mezclas mediante mezclado en fundido y se obtuvieron las películas mediante moldeo compresión. Se estudiaron sus propiedades mecánicas (fuerza de tensión al punto de fractura, módulo de elasticidad y deformación hasta el punto de fractura), de barrera (tasa de transmisión al vapor de agua, y tasa de transmisión a oxigeno) y ópticas (brillo y transmitancia interna). Como resultado, la resistencia a la tracción y rigidez del material se incrementaron con la incorporación de PLA y ESO, mientras que la capacidad de deformación se incrementó con la adición de ESO. Por otro lado, las propiedades de barrera contra el oxígeno estuvieron en el rango de requerimiento de envasado de diferentes grupos alimenticios y las propiedades de barrera al vapor de agua estuvieron por encima de los requerimientos de envasado alimenticio, aunque se mejoraron levemente con la incorporación de PLA y ESO. Por último, las propiedades ópticas mostraron poco brillo, siendo el comportamiento de materiales con superficie rugosa, la transparencia del TPS disminuyó con la incorporación de PLA y RH y aumento levemente con la incorporación de ESO.

Palabras clave: propiedades físicas, agente de interfase, material de refuerzo.

\section{Abstract}

Huge amount of lignocellulosic by-products is produced constantly in Colombia; most of which is burned andlor deposited on the ground. The aim of the present work was to study the development of composite materials based on thermoplastic starch (TPS), polylactic acid (PLA), rice husk (RH) and epoxidized soybean oil (ESO), as an alternative to RH 
Biocompuestos a base de almidón termoplástico, ácido poliláctico y cascarilla de arroz: efecto del aceite epoxidado de soya

utilization. Materials were blended by melt blending and films were elaborated by compression molding. Mechanical (tensile strength at breaking point, elastic and deformation modulus at breaking point), of barrier (transmission rate to water vapor and oxygen) and optical properties (gloss and internal transmittance) were studied. As a result, tensile strength and stifftness of materials increased with PLA and ESO incorporation, while deformation increased with ESO presence. On the other hand, barrier properties against oxygen were in the range of food packaging requirement and water vapor barrier values were higher than food packaging requirement, although showed a slight improvement with PLA and ESO incorporation. Finally, optical properties showed low gloss; this behavior is typical from rough surface materials, the TPS transparency decreased with PLA and RH presence and increased slightly with ESO incorporation.

Keywords: interface agent, physical properties, reinforcing materials.

\section{INTRODUCCIÓN}

A nivel mundial, la industria de arroz genera enormes cantidades de residuos ricos en lignocelulosa. Se estima una producción anual de arroz en grano superior a 600 millones de toneladas. A su vez, esto genera de 1 a 1,5 veces su peso en cascarilla, es decir, entre 600 y 900 millones de toneladas anuales (Karimi, Kheradmandinia \& Taherzadeh 2006). Estos residuos usualmente se desechan en el suelo, pero su alto contenido de minerales y baja tasa de degradación provocan daños en el medio ambiente. Otra práctica común es aprovecharlo como combustible, pero esto eleva la polución medioambiental y afecta la salud pública. Una alternativa viable es su aprovechamiento como componente de refuerzo de materiales poliméricos, generando biocomposites (Cano, et al. 2014; Dogossy \& Czigany 2011).

Una de las matrices poliméricas optativas para la incorporación de este tipo de materiales de refuerzo es el almidón termoplástico. Algunas de las ventajas de estos materiales es su bajo costo, alta barrera al oxígeno y buena procesabilidad mediante las técnicas convencionales como extrusión, moldeo compresión, inyección, entre otras (Ortega-Toro et al. 2014). Por otro lado, presentan deficiencias en cuanto a sus propiedades mecánicas, propiedades de barrera al vapor de agua y cambio de propiedades con el tiempo debido a la retrogradación del almidón. Algunos autores han estudiado el mejoramiento de estas propiedades funcionales mediante la mezcla con otros polímeros, como Poli (e-caprolactona) (PCL) (Ortega-Toro et al. 2015;
Mahieu, Terrié y Youssef 2015; Ortega-Toro et al. 2016), Ácido Poliláctico (PLA) (Wang et al. 2008), derivados de celulosa (Ma et al. 2008)y proteínas (Jiménez et al. 2012), entre otros. Entre las mezclas más prometedoras se encuentran aquellas realizadas con polímeros de carácter hidrofóbico como el PLA porque permite reducir la permeabilidad al vapor de agua y tiene un costo moderado en el mercado; sin embargo, las mezclas TPS/PLA no son del todo compatibles y necesitan de agentes que promuevan su afinidad química (Wang et al. 2008).

Los aceites epoxídicos constituyen una alternativa viable para proporcionarle al TPS algunos grupos funcionales que le permitan incrementar su afinidad química con el PLA, tal como lo han reportado otros autores (Xiong et al. 2013; Belhassen et al. 2014). Estos aceites son biodegradables, ambientalmente amigables y de alta disponibilidad. Concretamente, el aceite epoxidado de soja (ESO) tiene bajo costo, es derivado de fuentes renovables y es uno de los aceites epoxidados más usados, principalmente como aditivo del cloruro de polivinilo y como agentes de iniciación para producir polioles y espumas de poliuretano (Belhassen et al. 2014; Lin et al. 2008).

El objetivo de la presente investigación fue evaluar el comportamiento de materiales compuestos a base de una matriz polimérica de TPS-PLA con la incorporación de cascarilla de arroz como material de relleno y 
aceite epoxídico de soja para mejorar la afinidad química entre los componentes de la matriz y el agente de refuerzo.

\section{Materiales y MÉTODoS}

\section{A. Materiales}

El almidón de yuca fue adquirido de Almidones de Sucre (Almidones de Sucre S.A.S. Corozal, Colombia). El glicerol fue comprado en Alfaquímicos (Alfaquímicos S.A.S, Medellín, Colombia). El ácido poliláctico LL700 fue proporcionado por Químico Plásticos (Químico Plásticos, Envigado, Colombia). El aceite epoxídico de soja, grado B-22, fue proporcionado por la empresa Bretano Corp (Bretano Corp, Marvar, Bogotá-Colombia). La cascarilla de arroz fue suministrada por una finca productora ubicada en Ibagué (Colombia).

\section{B. Métodos}

\section{1) Preparación de los materiales compuestos}

Se mezcló manualmente almidón nativo, glicerol y agua en proporciones 1:0,3:0,5 respectivamente. Cada mezcla pesaba $100 \pm 0,1 \mathrm{~g}$. Posteriormente, se homogenizó mediante mezclado en fundido en una mezcladora de doble rodillo a $130^{\circ} \mathrm{C}$ y $10 \mathrm{rpm}$ durante 20 min, obteniendo almidón termoplástico (TPS). El TPS se trituró y se acondicionó durante 3 días a $0 \%$ de humedad relativa (H.R.) usando $\mathrm{P}_{2} \mathrm{O}_{5}$ para absorber la humedad. Los composites se prepararon mediante mezclado en fundido del TPS, PLA, fibras de cascarilla de arroz (RH) (tamaño $100 \mu \mathrm{m}$ ) y aceite epoxidado de soja (ESO), en las proporciones descritas en la Tabla 1. Estas proporciones fueron ajustadas mediante ensayos preliminares. Todos los componentes fueron secados previamente para evitar gritas en el material final. Se mezclaron a $160^{\circ} \mathrm{C}$ y $10 \mathrm{rpm}$ durante $30 \mathrm{~min}$. Posteriormente, se obtuvieron las películas mediante moldeo compresión a $160^{\circ} \mathrm{C}$ y $9 \mathrm{Kg} / \mathrm{cm}^{2}$ durante 5 min. Las películas fueron acondicionadas a $25{ }^{\circ} \mathrm{C}$ y $53 \%$ H.R. hasta alcanzar la humedad de equilibrio, previo a su caracterización. Se obtuvieron 10 películas de cada formulación.
Tabla 1.

Fracción másica $(\mathrm{Xi})$ de almidón, glicerol, PLA, ESO y cascarilla de arroz de las formulaciones estudiadas

\begin{tabular}{|c|c|c|c|c|c|}
\hline Formulación & $\mathbf{X}_{\text {almidón }}$ & $\mathbf{X}_{\text {glicerol }}$ & $\mathbf{X}_{\text {PLA }}$ & $\mathbf{X}_{\text {ESO }}$ & $\mathbf{X}_{\mathrm{RH}}$ \\
\hline F1 & 0,769 & 0,231 & & & \\
\hline F2 & 0,667 & 0,200 & 0,133 & & \\
\hline F3 & 0,656 & 0,197 & 0,131 & 0,016 & \\
\hline F4 & 0,645 & 0,194 & 0,129 & 0,032 & \\
\hline F5 & 0,645 & 0,194 & 0,129 & & 0,032 \\
\hline F6 & 0,625 & 0,188 & 0,125 & 0,031 & 0,031 \\
\hline
\end{tabular}

\section{2) Caracterización de las películas}

\section{1) Propiedades mecánicas}

La fuerza de tensión máxima en el punto de fractura (TS), deformación máxima en el punto de fractura (E) y módulo de elasticidad (EM) de las películas fueron determinadas con una prensa universal de ensayos (TA.XT plus texture analyser) de acuerdo al método estándar ASTM D882 (Astm 2001). Se usaron probetas de 2,5 por $10 \mathrm{~cm}$ con una velocidad de $50 \mathrm{~mm} \mathrm{~min}^{-1}$ hasta su fractura. Se hicieron 10 repeticiones para cada formulación.

\section{2) Propiedades de barrera}

La permeabilidad al vapor de agua (WVP) fue determinada siguiendo el método estándar ASTM E96-95 (Astm 1995) con algunas modificaciones descritas en trabajos anteriores (Ortega-Toro et al. 2014), se utilizó un gradiente de humedades de $53 \%$ H.R. a $100 \%$ a $25^{\circ} \mathrm{C}$. La permeabilidad al oxígeno (OP) fue determinada siguiendo el método estándar ASTM D3985-95 (Astm 2002), se llevó a cabo a $53 \%$ H.R. y $25^{\circ} \mathrm{C}$ usando un equipo OX-TRAN ${ }^{\circledR}$ (Model 2/21 series MOCON INC). Ambas pruebas se realizaron por triplicado.

\section{3) Propiedades ópticas}

La transmitancia interna $(\mathrm{Ti})$ de las películas fue determinada en un espectrofotómetro UV-VIS (Model 100707 - Merck Millipore) en películas de 1 x $3 \mathrm{~cm}$ equilibradas a $25^{\circ} \mathrm{C}$ y $53 \%$ H.R. El rango de longitudes de onda medidas fue entre 200 y $1000 \mathrm{~nm}$. Se tuvieron en cuenta los valores de Ti a $450 \mathrm{~nm}$ para su 
análisis. El brillo de las películas fue determinado a un ángulo de incidencia de $85^{\circ}$ mediante un brillometro (PCE-GM 60) siguiendo el método estándar ASTM D523 (Astm 1999). Las medidas de propiedades ópticas fueron determinadas por triplicado.

El análisis estadístico fue realizado con Statgraphics Plus para Windows (Manugistics Corp., Rockville, MD) mediante un análisis de varianza (Anova) y la prueba de mínimas diferencias significativas de Fisher con un nivel de confianza del $95 \%$.

\section{RESULTADOS Y DISCUSIÓN}

Todos los resultados presentaron una distribución normal. En la Fig. 1 se puede observar el comportamiento mecánico (EM: Modulo Elástico; TS: Fuerza de tensión máxima en el punto de fractura; E: deformación máxima en el punto de fractura) de las formulaciones estudiadas acondicionadas a 53\% de H.R.

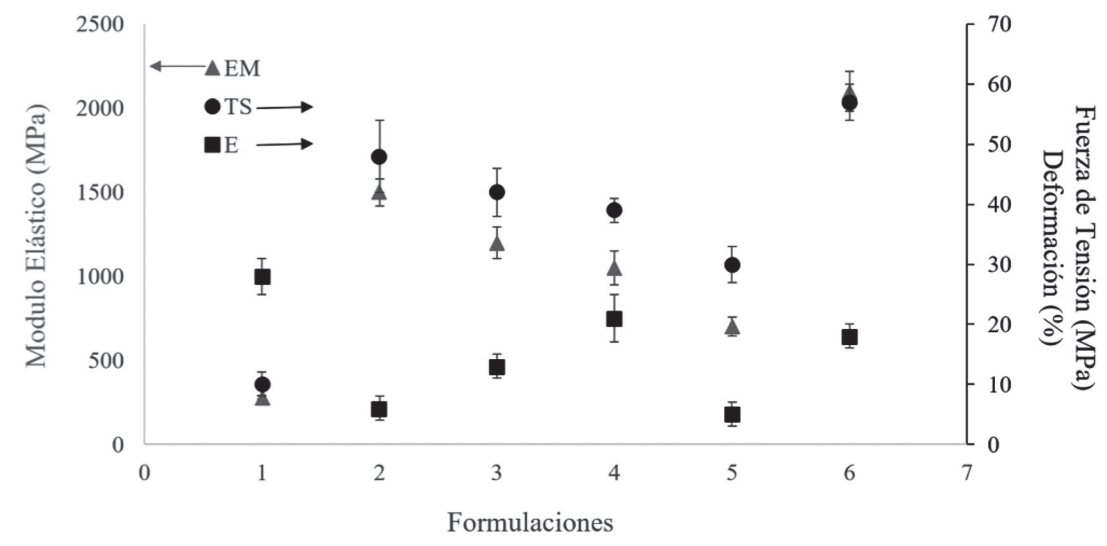

Fig. 1 Propiedades mecánicas (EM: Modulo Elástico; TS: Fuerza de tensión máxima en el punto de fractura; E: deformación máxima en el punto de fractura) de las formulaciones estudiadas acondicionadas a $53 \%$ de H.R. Las barras indican limites LSD con 95\% de confianza.

Se observa el efecto que ejercen los diferentes componentes y sus combinaciones sobre el almidón termoplástico. La adición de PLA al TPS provoca la disminución de capacidad de deformación del material y el aumento de su resistencia y rigidez. El aceite epoxidado de soya ańadido en dos concentraciones incrementa significativamente la capacidad de deformación y disminuye, de forma menos marcada, tanto la rigidez como la resistencia del material, esto se puede atribuir al carácter plastificante del aceite, que a la vez parece mejorar la afinidad entre el TPS y el PLA. Cuando se añadió la cascarilla de arroz a la mezcla TPS/PLA (F5), todas las propiedades mecánicas se disminuyeron drásticamente.

Esto fue causado por la baja interacción entre los componentes de la matriz polimérica (TPS-PLA) y el material de refuerzo. Posteriormente, cuando se añadió el ESO a la matriz polimérica que contenía la cascarilla (F6), se observa como aumentan significativamente la capacidad de deformación del material, su resistencia a la tracción y su rigidez. Este cambio en las propiedades pone de manifiesto que el ESO puede actuar como un buen agente de inter-fase en material compuesto TPS/PLA y cascarilla de arroz.

Por otro lado, en la Fig. 2 se muestra un mapa de propiedades de barrera. Se observa la tasa de transmisión del vapor de agua (WVTR) y la tasa de transmisión del oxígeno (OTR) de las formulaciones estudiadas acondicionadas a 53\% H.R. Además, se muestra los requerimientos de propiedades de barrera de diferentes grupos alimenticios (rectángulo grande sombreado: salsas, snacks, nueces, leche UHT, café, infusiones, comida para bebé, aceites; rectángulo pequeño sombreado: productos cárnicos y envases de 
atmosferas modificadas) y algunos polímeros usados en la industria alimentaria como EVOH, PET y PLA (Schmid et al. 2012).

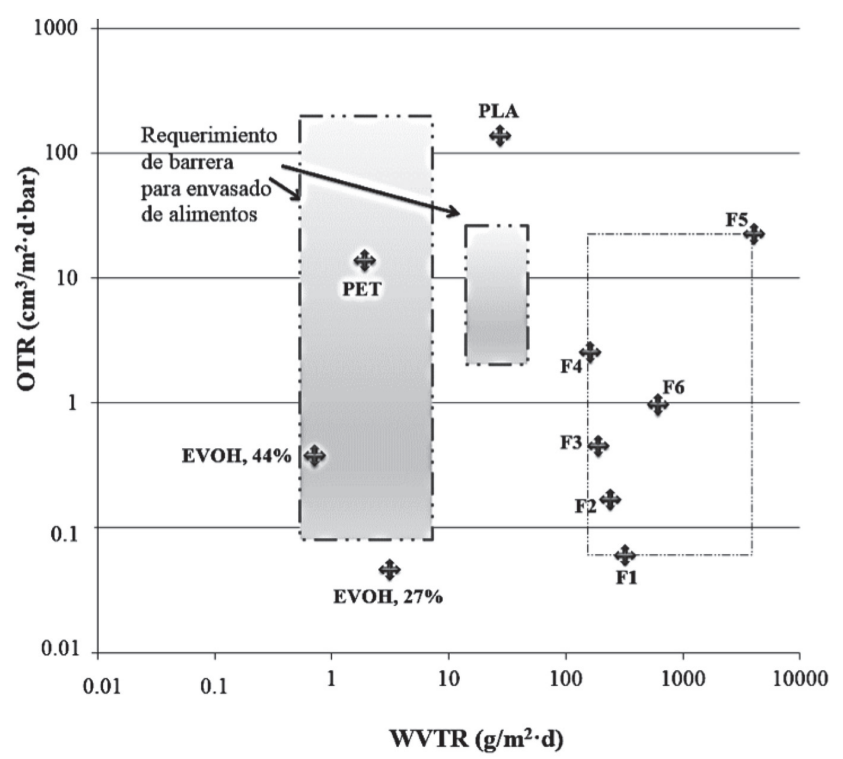

Fig. 2 Propiedades de barrera al oxigeno (OTR: transmisión de oxígeno) y al vapor de agua (WVTR: transmisión de vapor de agua) de las formulaciones estudiadas acondicionadas a $25^{\circ} \mathrm{C}$ y 53\% de H.R. Requerimientos de barrera de algunos alimentos (rectángulos sombreados) y algunos polímeros usados en la industria alimentaria.

Los resultados muestran que todas las formulaciones estudiadas tienen propiedades de barrara al oxígeno adecuadas para aplicaciones alimentarias, especialmente el TPS (F1) estando al mismo nivel de OTR que el EVOH (27\% de etileno). Cuando se ańade el PLA a la formulación (F2) y el ESO (F3 y F4) se observa la disminución progresiva de WVTR y el aumento de OTR, de acuerdo al carácter hidrofóbico de ambos componentes. Posteriormente, cuando se añade la fibra de arroz a la matriz polimérica (F5) se observa un detrimento marcado de las propiedades de barrera, debido a la baja interacción entre sus componentes y que posiblemente se crean micro poros en la matriz, facilitando el transporte de masa mediante capilaridad. Finalmente, la presencia de ESO sobre la matriz del material compuesto mostró la capacidad de reducir ambas tasas de transmisión, en concordancia con su capacidad para aumentar las interacciones entre los componentes de la matriz y a su carácter hidrofóbico, así como la tortuosidad a la difusión de los agentes permeantes a través de la matriz del composite.

Como lo muestran los resultados, estas formulaciones tienen propiedades de barrera al oxígeno adecuadas para aplicaciones alimentarias, pero sus propiedades de barrera al vapor de agua son deficientes. Por lo anterior, se podría pensar en envases multicapa, como estrategia para el mejoramiento de las propiedades de barrera, donde una de las capas sea una formulación rica en almidón termoplástico (barrera contra el oxígeno) y otra capa sea un polímero de alta barrera al vapor de agua. Estos materiales han sido estudiados últimamente por diferentes autores como (OrtegaToro et al.2015) quienes estandarizaron la obtención de películas bicapa combinando capas de TPS y PCL; (Ferreira et al.2016) desarrollaron y caracterizaron un material bicapa de Fucopol y quitosano; Hosseini et al. (2016) estudiaron las propiedades de barrera de películas multicapa a base de PLA y gelatina de pescado y (Mahieu et al.2017) estudiaron el papel del ácido ascórbico y hierro como absorbedores de oxígeno en películas multicapa de TPS y PCL.

Finalmente se caracterizaron las propiedades ópticas de las películas. En la Tabla 2 se muestra los valores porcentuales de brillo a un ángulo de incidencia de $85^{\circ}$ y la transmitancia interna a una longitud de onda de $450 \mathrm{~nm}$ para las películas estudiadas acondicionadas a $53 \%$ de H.R.

Tabla 2.

Valores promedio y desviación estándar del brillo $\left(85^{\circ}\right)$ y transmitancia interna $-\mathrm{Ti}-(450 \mathrm{~nm})$ de las formulaciones estudiadas acondicionadas a $25^{\circ} \mathrm{C}$ y $53 \%$ de H.R.

\begin{tabular}{|c|c|c|}
\hline Formulación & Brillo $\left(\mathbf{8 5} 5^{\circ}\right)$ & TI (450 NM) \\
\hline F1 & $41(2)^{\mathrm{A}}$ & $86,5(0,5)^{\mathrm{A}}$ \\
\hline F2 & $30(3)^{\mathrm{BC}}$ & $79,2(0,3)^{\mathrm{B}}$ \\
\hline F3 & $33(2)^{\mathrm{B}}$ & $86,4(0,4)^{\mathrm{A}}$ \\
\hline F4 & $34(2)^{\mathrm{B}}$ & $87,3(0,7)^{\mathrm{A}}$ \\
\hline F5 & $25(2)^{\mathrm{C}}$ & $77,2(0,5)^{\mathrm{C}}$ \\
\hline F6 & $28(2)^{\mathrm{C}}$ & $77,5(0,4)^{\mathrm{C}}$ \\
\hline
\end{tabular}


Letras superíndices diferentes dentro de la misma columna indican diferencia significativa entre las formulaciones $(\mathrm{p}<0,05)$.

En general se observa que los materiales estudiados tienen poco brillo, oscilan entre valores de 28 (F6) a 41 (F1), siendo característico de este tipo de materiales. Esta propiedad tiene una relación directa con la topografía superficial de las películas. Si las películas tienen una superficie rugosa darán por resultado películas de poco brillo a causa de la reflexión difusa del haz de luz incidente; por el contrario, si las películas son lisas reflejarán el haz de luz de forma especular dando por resultado altos valores de brillo. En cuanto a la transmitancia interna de las películas, este parámetro tiene relación con la homogeneidad del material, entre más altos sean sus valores, más homogénea será el material. Se puede inferir que el PLA disminuye significativamente la transparencia de TPS, mientras que la adición de ESO incrementa la transparencia de las mezclas TPS/PLA. Por otro lado, como es de esperar, la adición de un material de relleno a la mezcla TPS/ PLA disminuye su transparencia.

\section{Conclusiones}

Se elaboraron materiales a base de almidón termoplástico (TPS), ácido poliláctico (PLA), cascarilla de arroz $(\mathrm{RH})$ y aceite epoxídico de soya (ESO) mediante mezclado en fundido y moldeo compresión. Las propiedades mecánicas mostraron incremento de la rigidez-resistencia y disminución de la capacidad de deformación con la incorporación de PLA al TPS, la incorporación del ESO a la mezcla TPS/PLA causó la disminución de la rigidez-resistencia y aumento de la capacidad de deformación, mientras que la adición del ESO al material compuesto TPS/PLA/RH mostró el incremento de todas las propiedades mecánicas estudiadas. En este sentido es conveniente el uso de ESO para mejorar las propiedades mecánicas de las mezclas poliméricas y materiales compuestos. En cuanto a las propiedades de barrera, todas las formulaciones mostraron buenas propiedades de barrera al oxígeno estando dentro del rango de los requerimientos de barrera al oxígeno de diferentes grupos alimenticios. Sin embargo, las propiedades de barrera al vapor de agua fueron demasiado altas para aplicaciones de envasado alimentario, podrían mejorarse mediante el uso de otras estrategias como la formación de bicapas. Aunque se observó diminución de la permeabilidad al vapor de agua cuando se añadió ESO a las mezclas poliméricas. Para finalizar, las propiedades ópticas mostraron el comportamiento típico de materiales de superficie rugosa y la transparencia de las películas de TPS se disminuyeron cuando se incorporaron PLA y RH, y aumento levemente cuando se incorporó ESO.

\section{Agradecimientos}

El autor agradece a la Universidad Nacional Abierta y a Distancia por el apoyo mediante el proyecto interno PIE 17 - 07.

\section{REFERENCIAS}

Astm 1995, 'Standard test methods for water vapour transmission of materials. Standard designations: E96e95', en Astm, Annual book of Astm standards, American Society for Testing and Materials, Philadelphia.

Astm. 1999. 'Standard test method for specular gloss. Standard designations: D523', en Astm, Annual book of Astm standards, American Society for Testing and Materials, Philadelphia.

Astm 2001, 'Standard test method for tensile properties of thin plastic sheeting. Standard designations: D882', en Astm, Annual book of Astm standards, American Society for Testing and Materials, Philadelphia.

Astm 2002, 'Standard test method for oxygen gas transmission rate through plastic film and sheeting using a coulometric sensor', en Astm, Annual book of Astm standards, American Society for Testing and Materials, Philadelphia.

Belhassen, R, Vilaseca, F, Mutjé, P \& Boufi, S 2014, 'Thermoplasticized starch modified by reactive blending with epoxidized soybean oil', Industrial Crops and Products, 53, 261-267.

Cano, A, Jiménez, A, Cháfer, M, Gonzalez C \& Chiralt, A 2014, 'Effect of amylose: amylopectin ratio and rice bran addition on starch films properties', Carbohydrate Polymers, 111, 543-555.

Dogossy, G \& Czigany, T 2011, 'Thermoplastic starch composites reinforced by agricultural by-products: properties, biodegradability, and application', Journal of Reinforced Plastics and Composites, 30, 1819-1825. 
Ferreira, A, Torres, C, Freitas, F, Sevrin, C, Grandfils, C, Reis, M, Alves, V \& Coelhoso, M 2016, 'Development and characterization of bilayer films of FucoPol and chitosan', Carbohydrate Polymers, 147, 8-15.

Jiménez, A, Fabra, M, Talens, P \&. Chiralt, A 2012, 'Effect of sodium caseinate on properties and ageing behaviour of corn starch based films' Food Hydrocolloids, 29, 265-271.

Hosseini, S, Javidi, Z \& Rezaei, M 2016, 'Efficient gas barrier properties of multi-layer films based on poly(lactic acid) and fish gelatin', International Journal of Biological Macromolecules, 92, 1205-1214.

Karimi, K, Kheradmandinia S \& Taherzadeh, M 2006, 'Conversion of rice straw to sugars by dilute-acid hydrolysis', Biomass and Bioenergy, 30, 247-253.

Lin, B, Yang, L, Dai, H \& Yi, A 2008, 'Kinetic studies on oxirane cleavage of epoxidized soybean oil by methanol and characterization of polyols', Journal of American Oil Chemestry Society, 85, 113-117.

Ma, X, Chang, P \& Yu, J 2008, 'Properties of biodegradable thermoplastic pea starch/carboxymethyl cellulose and pea starch/ microcrystalline cellulose composites', Carbohydrate Polymers, $72,369-375$.

Mahieu, A, Terrié, C \& Youssef, B 2015, 'Thermoplastic starch films and thermoplastic starch/polycaprolactone blends with oxygen-scavenging properties: Influence of water content', Industrial Crops and Products, 72, 192-199.

Mahieu, A, Terrié, C \& Leblanc, N 2017, 'Research Article. Role of ascorbic acid and iron in mechanical and oxygen absorption properties of starch and polycaprolactone multilayer film', Packaging Research, 2, 1-11.
Ortega-Toro, R, Jiménez, A, Talens, P \& Chiralt, A 2014, Properties of starch-hydroxypropyl methylcellulose based films obtained by compression molding', Carbohydrate Polymers, 109, pp. 155-165.

Ortega-Toro, R, Morey, I, Talens, P \& Chiralt, A 2015, 'Active bilayer films of thermoplastic starch and polycaprolactone obtained by compression molding', Carbohydrate polymers, 127, 282-290.

Ortega-Toro, R, Muñoz, A, Talens, P \& Chiralt, A 2015, 'Improvement of properties of glycerol plasticized starch films by blending with a low ratio of polycaprolactone and/or polyethylene glycol', Food Hydrocolloids, 56, 9-19.

Ortega-Toro, R, Contreras, J, Talens P, \& Chiralt, A 2016, 'Physi$\mathrm{cal}$ and structural properties and thermal behaviour of starchpoly (E-caprolactone) blend films for food packaging', Food Packaging and Shelf Life, 5, 10-20.

Schmid, M, Dallmann, K, Bugnicourt, E, Cordoni, D, Wild, F, Lazzeri, A \& Noller, K 2012, 'Properties of whey-proteincoated films and laminates as novel recyclable food packaging materi-als with excellent barrier properties', International Journal of Polymer Science, 8, -7.

Wang, N, Yu, J, Chang, P \& Ma, X 2008, 'Influence of formamide and water on the properties of thermoplastic starch/poly(lactic acid) blends', Carbohydrate Polymers, 71, 109-118.

Xiong, Z, Yang, Y, Feng, J, Zhang, X Zhang, C, Tang, Z \& Zhu, J 2013, 'Preparation and characterization of poly(lactic acid)/ starch composites toughened with epoxidized soybean oil', Carbohydrate Polymers, 92, 810-816. 
